\title{
Column agglutination assay using polystyrene microbeads for rapid detection of antibodies against SARS-CoV-2
}

Vidhishri Kesarwani ${ }^{1,2,3^{*}}$, Julia A. Walker ${ }^{1,2,3^{*}}$, Edward C. Henderson ${ }^{1,2,3}$, Gabriel Huynh $^{1,2,3}$, Heather McLiesh ${ }^{1,2}$ Maryza Graham ${ }^{4}$, Megan Wieringa ${ }^{4}$, Mark M. Banaszak Holl ${ }^{1,2}$, Gil Garnier ${ }^{1,2}$ and Simon R. Corrie ${ }^{1,2,3}$

*joint first authors

${ }^{1}$ Department of Chemical and Biological Engineering, Monash University, Clayton, Victoria 3800, Australia

2 Bioresource Processing Research Institute of Australia (BioPRIA), Monash University, Clayton, Victoria 3800

${ }^{3}$ Centre to Impact AMR, Monash University, Clayton, Victoria 3800, Australia

${ }^{4}$ Department of Microbiology and Monash Infectious Diseases, Monash Health, Clayton, Victoria 3168, Australia; Department of Clinical Sciences, Monash University, Clayton, Victoria 3168, Australia

Corresponding author: simon.corrie@monash.edu 


\section{Supplementary Figures}

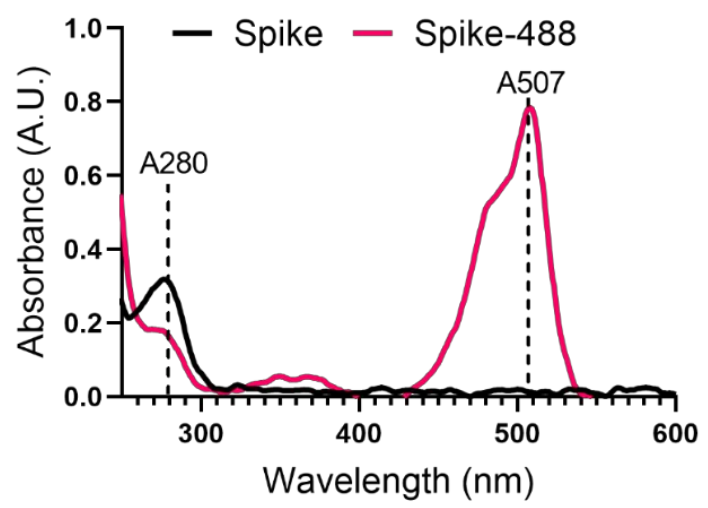

Supplementary Figure 1. UV-Vis of recombinant SARS-CoV-2 spike protein with and without labelling with BDFL-488. The fluorescent label was chemically functionalised on the recombinant protein through EDC/NHS bioconjugation chemistry. The peak absorbance observed at $507 \mathrm{~nm}$ is attributed to the attachment of BDFL-488 on the spike protein.

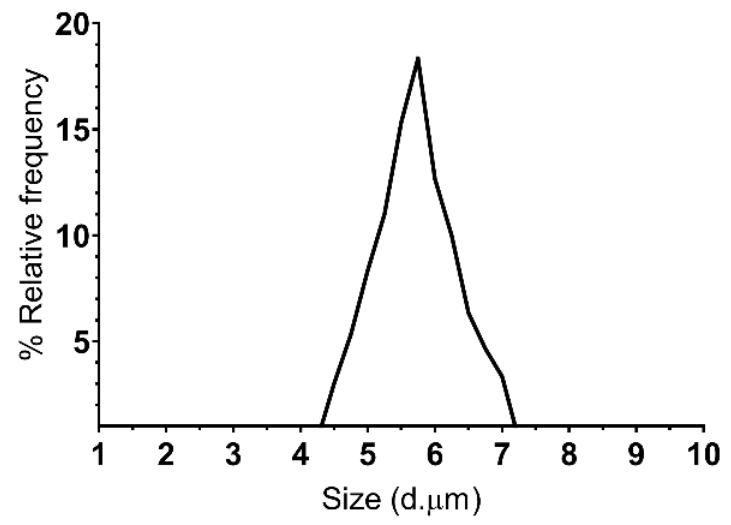

Supplementary Figure 2. Particle size distribution of PS-Control microbeads. Particle size measurements were obtained using optical microscopy.

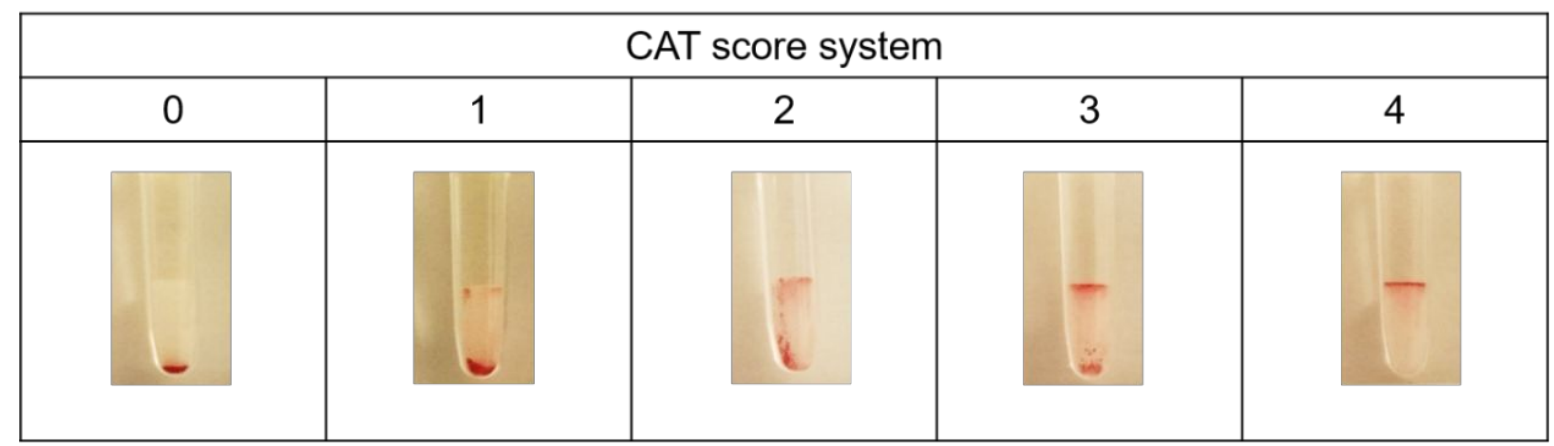

Supplementary Figure 1. CAT scoring system of microbeads adapted from the CAT scoring system employed in blood typing using erythrocytes ${ }^{1}$. 


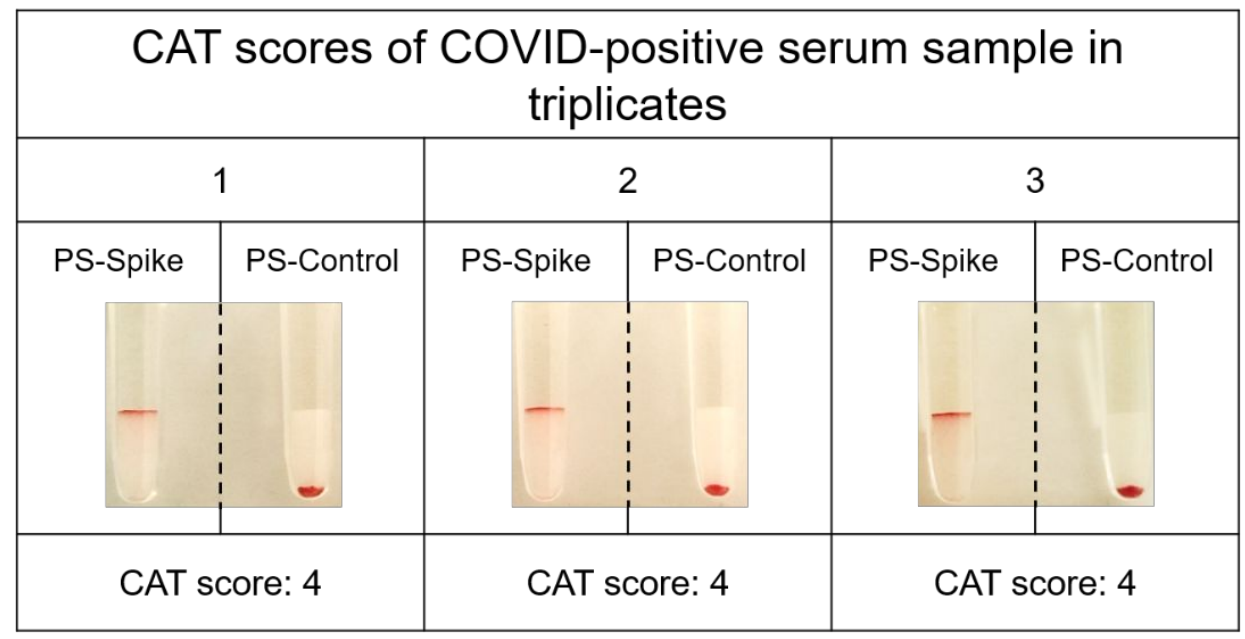

Supplementary Figure 4. CAT scores of COVID-19 positive serum sample run in triplicates, were performed 6 months after the experiment shown in Figure 6. Both PS-Spike and PS-Control show yield the same CAT score when run in triplicates.

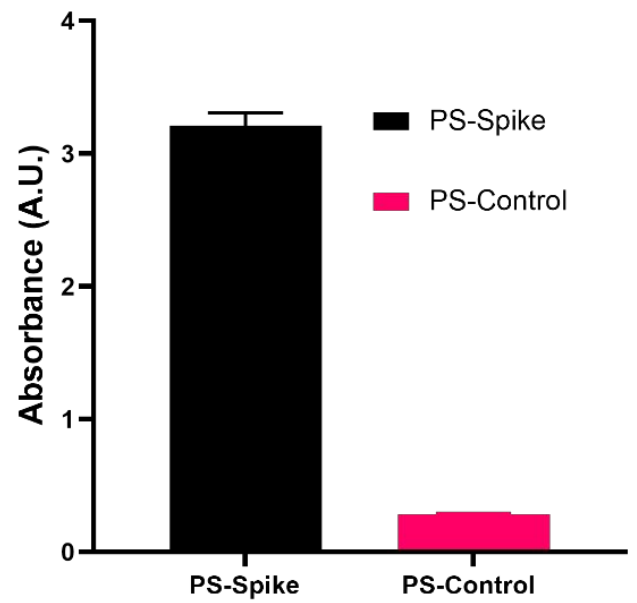

Supplementary Figure 5. Validation of Spike protein function on PS particle when incubated with a polyclonal, Anti-Spike (Sino Biological) 6 months after bioconjugation via passive absorption performed on PS microbeads. 


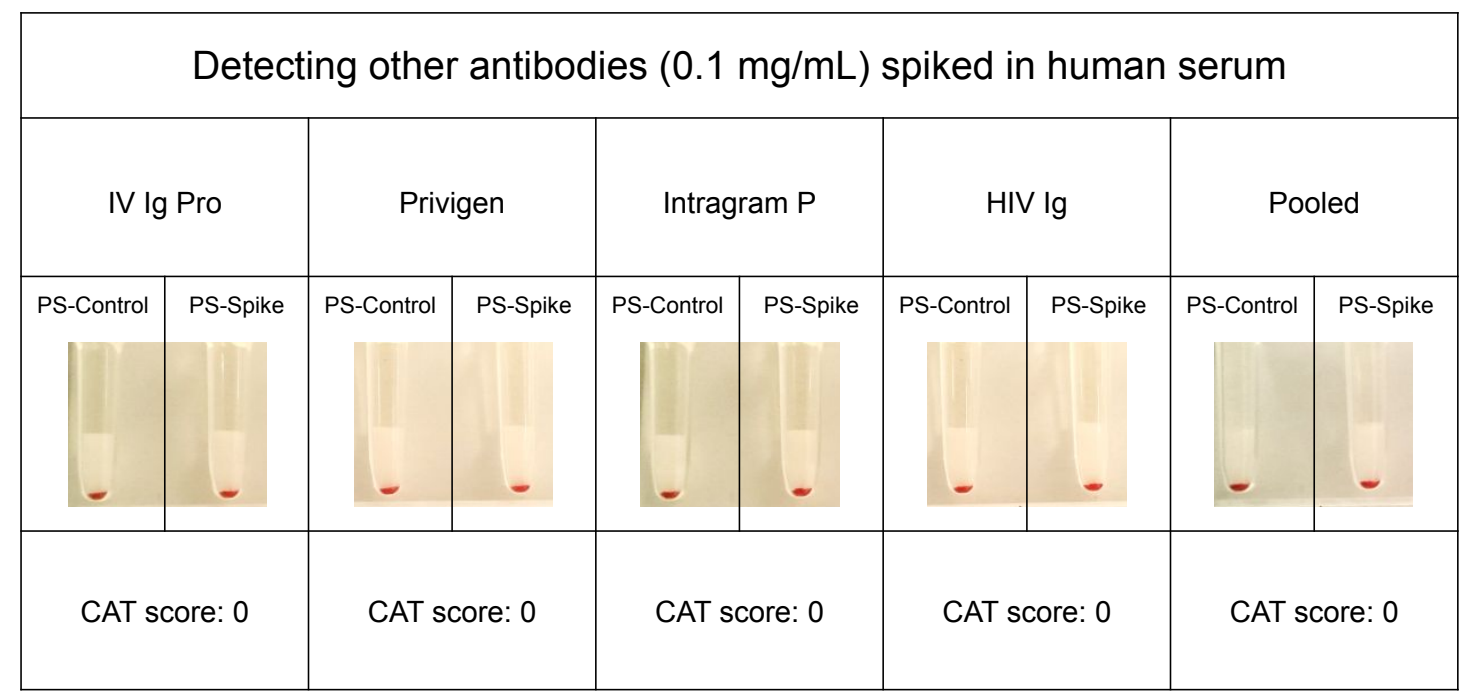

Supplementary Figure 6: CAT scores of PS-Control and PS-spike beads incubated with normal human serum spiked with $0.1 \mathrm{mg} / \mathrm{mL}$ polyclonal Ig samples. IV Ig Pro, Privigen and Intra P are commercial IV-Ig products (CSL Ltd) collected and purified from human blood donors for use as therapies for a variety of disorders. HIV-Ig is prepared from pooled plasma of asymptomatic HIV antibody-positive donors, obtained through the NIH HIV Reagent Program, Division of AIDS, NIAID, $\mathrm{NIH}$ : Polyclonal Anti-Human Immunodeficiency Virus Immune Globulin, Pooled Inactivated Human Sera, ARP-3957, contributed by NABI and National Heart Lung and Blood Institute (Dr. Luiz Barbosa). These samples were generously provided by Prof Stephen Kent and Dr Adam Wheatley.

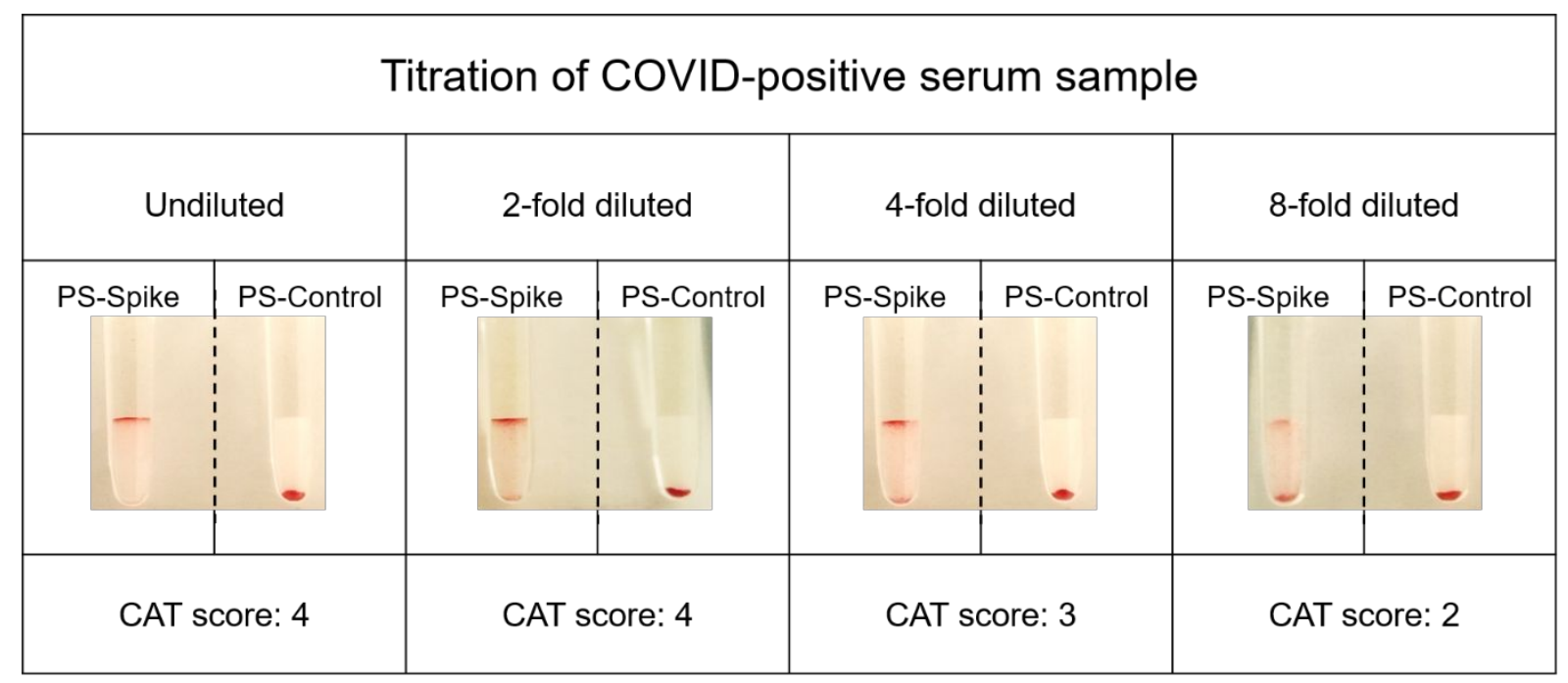

Supplementary Figure 7. Titration of COVID-19 positive serum and its respective CAT scores. Titrating COVID-positive serum till 8-fold dilution yields gel card scores $>2$. 


\begin{tabular}{|c|c|c|c|}
\hline \multicolumn{2}{|c|}{ EDTA Plasma Samples } & \multicolumn{2}{|c|}{ Heparin Plasma Samples } \\
\hline \multicolumn{2}{|c|}{} & \multicolumn{2}{|c|}{} \\
\hline $\begin{array}{c}\text { PS-Control } \\
\text { microbeads }\end{array}$ & $\begin{array}{c}\text { PS-Spike } \\
\text { microbeads }\end{array}$ & $\begin{array}{c}\text { PS-Control } \\
\text { microbeads }\end{array}$ & $\begin{array}{c}\text { PS-Spike } \\
\text { microbeads }\end{array}$ \\
\hline
\end{tabular}

Supplementary Figure 8. CAT scores of PS-Control and PS-Spike microbeads treated with EDTA plasma and Heparin plasma samples obtained from uninfected and unvaccinated donors. The CAT scores of PS-Control and PS-Spike when treated with EDTA plasma samples yield a CAT score of 4 , while PS-Control and PS-Spike beads treated with Heparin plasma yields a score of 3 and 4 , respectively.

\section{References}

(1) Reis, K. J.; Chachowski, R.; Cupido, A.; Davies, D.; Jakway, J.; Setcavage, T. M. Column agglutination technology: the antiglobulin test. Transfusion 1993, 33 (8), 639-643, DOI:

https://doi.org/10.1046/j.1537-2995.1993.33893342744.x. 